\title{
A Conservation of Resources Approach to Blackberry Use, Work-Family Conflict and Well-Being: Job Control and Psychological Detachment from Work as Potential Mediators
}

\author{
Sophie Ward ${ }^{1} \&$ Gail Steptoe-Warren ${ }^{1}$ \\ ${ }^{1}$ Faculty of Health and Life Sciences, Psychology and Behavioural Sciences Department, Coventry University, \\ Coventry, UK
}

Correspondence: Sophie Ward, Faculty of Health and Life Sciences, Psychology and Behavioural Sciences Department, Coventry University, Coventry, CV1 5FB, UK. E-mail: sophie.ward@coventry.ac.uk

Received: November 9, 2013 Accepted: November 30, 2013 Online Published: December 16, 2013

doi:10.5539/emr.v3n1p8

URL: http://dx.doi.org/10.5539/emr.v3n1p8

\begin{abstract}
This paper provides a comparison study of the relationships between using the Blackberry (BB) device for work purposes during non-work hours and (a) work-family conflict and (b) well-being, and the potential mediators of these relationships from a Conservation of Resources (COR) approach. Findings revealed that greater usage of the $\mathrm{BB}$ for work purposes during non-work hours was related to higher levels of work-family conflict. In particular greater frequency of use was related to an inability to psychologically detach from work, resulting in work-family conflict and low levels of well-being. Furthermore using the device for long periods was also related to an inability to psychologically detach from work, resulting in low levels of well-being. The findings did not support job control as a potential mediator; although support was found for the COR theory, as the mediating effect of detachment from work was greater than the mediating effect of job control on the relationship between BB use and work-family conflict, supporting that resource loss is more salient than resource gain. In light of these findings this paper proposes that organisations should aim to reduce the negative impact that using this device for work purposes during non-work hours has on employees, by either reducing the usage of the device or by ensuring employees are capable of handling the device and demands of the job.
\end{abstract}

Keywords: Blackberry, communication technology, conservation of resources theory, perceived job control, psychological detachment from work, work-family conflict, well-being

\section{Introduction}

\subsection{Communication Technologies within Organisations: Work-Family Conflict and Well-Being}

Communication technologies have for a long time impacted upon working norms, resulting in more flexible contracts and working hours, and enabling an increase in global communication and outsourcing which has been essential in such a highly competitive global market (Vendramin et al., 2000). Over time the penetration of new communication technologies such as smart-phones which has been fast paced rising 12\% from 2011 (Ofcom, 2012), has resulted in work tasks no longer being primarily conducted in the office on a fixed computer, but to being location and time independent (Price, 2010). There has therefore been a disappearance of the "traditional boundaries of work' that used to be prescribed, (Khanna \& Randolph, 2008) as employees are no longer bound by traditional geographic and temporal constraints, instead they can stay connected to the workplace, and work anytime and anywhere rather than at their place of work or during their contracted work hours (O'Driscoll, Brough, \& Kalliath, 2006).

The ability to stay connected and monitor incoming content has been found to be detrimental to employees, resulting in compulsive checking, dependence and an inability to mentally switch off from work (Mazmanian, Orlikowski, \& Yates, 2006). This leads to work demands interfering with the family role causing work-family conflict, as both work and family domains become 'mutually incompatible'(Greenhaus \& Beutell, 1985). Furthermore conflict in these major roles decreases an employee's level of life satisfaction (Carlson \& Perrewé, 1999), increases psychological distress, somatic complaints (Frone, Russell, \& Cooper, 1992, p. 197), and intensifies both exhaustion and disengagement (Karatepe, Sokmen, Yavas, \& Babakus, 2010). It can be suggested that these new communication technologies have a negative impact upon employees, and 
consequently the usage of these devices should be reduced. It can be argued from a human agency perspective that these devices do not force the user to stay connected, it is the user's choice as to how they take on, negotiate and accept the technology (Silverstone \& Mansell, 1996). Therefore the blame cannot be attributed to the technology devices alone. Indeed these new communication technologies can also benefit employees as they enable them greater flexibility as to when and where they do their work, which increases their personal control (Bélanger, 1999) and decreases stress related to balancing work and family needs (Hill, Hawkins, Ferris, \& Weitzman, 2001).

There are both positive and negative outcomes associated with the use of mobile communication technologies within organisations, and it is suggested that employees face a contradiction; they enjoy the flexibility and control mobile communication technologies provide but they spend less time disengaged from work as a consequence (Frissen, 2000). This contradiction has been defined by Jarvenpaa and Lang (2005) as the Empowerment/ Enslavement Paradox; whilst mobile communication technologies empower the user with a range of choices including location, they prevent them from creating and maintaining communicative distance from others. To date there is little research which provides an adequate theoretical explanation for this paradox, with the exception of the research of Richardson and Thompson (2012), which utilised the COR theory (Hobfoll, $1988,1989,2001)$ to explain this paradox.

\subsection{The Conservation of Resources (COR) Theory: Resource Loss vs. Resource Gain}

The COR theory propounded by Hobfoll (1989) suggests that people actively seek to obtain, build and protect their resources, including, objects, personal characteristics, conditions and energies. If an individual's resources are depleted or threatened, or if they fail to gain resources after resource investment then psychological stress occurs (Hobfoll, 1988, 1989, 2001). Research has shown that a great threat to resources is high work demands (Schaufeli \& Bakker, 2004), including role ambiguity, work pressure and work load (Lee \& Ashforth, 1996). Over time increased exposure to high work demands results in excessive depletion of energetic and social resources, a process which Freudenberger (1974) defines as burnout, a psychological syndrome involving emotional exhaustion, depersonalisation, and reduced personal accomplishment (Maslach, 1982).

To reduce burnout and aid stress resistance it is essential that there is an interruption of loss spirals and activation of gain spirals (Hobfoll \& Freedy, 1993). One way in which a gain spiral may occur is through time away from work (Eden, 2001). If an employee spends their time away from work thinking about work or engaging in work-related activities such as taking job-related calls they will not experience stress relief, and it will have a negative effect on their well-being (Sonnentag, 2001) and may cause work-family conflict (Illies et al., 2007). If during time away from work an employee is able to 'mentally switch off' from work-related duties, it will allow for their depleted personal resources to be replenished (Westman \& Eden, 1997) and increase their well-being (Sonnentag \& Bayer, 2005). This has been supported in a variety of respite studies that have investigated detachment from work due to a single day off (Frankenhaeuser et al., 1989), vacations (Westman \& Eden, 1997), and computer shutdowns (Eden, 1990).

In addition the possession of personal resources such as perceived job control can also help to interrupt loss spirals (Braunstein-Bercovitz, Frish-Burstein, \& Benjamin, 2012). It is suggested by a variety of researchers that individuals are motivated to seek control over their environment as high levels of perceived job control can aid effective coping in stressful situations, increase well-being (Thompson \& Prottas, 2006) and job satisfaction (Ross \& Reskin, 1992), and reduce fatigue (Van Yperen \& Hagedoorn, 2003), emotional exhaustion (De Jonge, Janseen, \& Van Breukelen, 1996), turnover intentions and work-family conflict (Kossek, Lautsch, \& Eaton, 2006).

It is, therefore, important that individuals invest resources to gain additional resources and protect against resource loss (Hobfoll, 1998, pp. 73-75). However, although employees can attempt to minimise their loss of resources, it has been suggested by Freedy and Hobfoll (1994) that work demands use up an employee's resources quicker than the resources are replenished. This has been supported in a meta-analysis on the correlates of burnout, which showed that over time individuals are affected more by chronic resource demands than resource gains (Lee \& Ashforth, 1996). This relates to the key principle of the COR theory outlined by Hobfoll (1989), that the impact of resource loss far outweighs the impact of equivalent resource gain.

\subsection{The Research of Richardson and Thompson (2012)}

Based on past literature and the COR theory principles it was proposed by Richardson and Thompson (2012, p. 30 ) that employees may continue to use their devices for work purposes during non-work hours as they believe it helps them to build or conserve valuable resources related to their work, such as the ability to keep on top of work demands. This in turn gives them a greater perception of control (resource gain) which can be beneficial in 
protecting against work-family conflict and increase well-being. By using the devices for work purposes during non-work hour's employees are remaining continuously connected to the workplace, which impedes their ability to psychologically detach from work (resource loss) and replenish lost resources which in turn may increase work-family conflict and decrease well-being (Richardson \& Thompson 2012, pp. 30-31).

The findings of Richardson and Thompson $(2012$, p. 29) revealed that greater mobile technology use during time away from work was directly related to higher levels of work-family conflict. Moreover technology use was positively related to job control and negatively related to detachment from work, and both variables in turn were negatively related to work-family conflict supporting past literature. Furthermore the mediating effect of detachment from work on the relationship between technology use and work-family conflict was greater than the mediating effect of job control, supporting the COR theory principle that resource loss is more salient than resource gain (Hobfoll, 1989). The findings did not support the relationship between greater mobile technology use during time away from work and a decrease in well-being, although Richardson and Thompson (2012) acknowledged this might have been due to the relatively young sample (mean age 34.3) used, as younger employees are more likely to be comfortable with using technology (Harrison \& Rainer, 1992).

\subsection{A Lack of Generalisability of the Research Findings}

Despite the addition to knowledge that Richardson and Thompson's (2012) findings have provided the results are not generalisable across other countries, as the study was conducted on a large media organisation in the north-eastern US. The work-family attitudes of individuals vary across different countries resulting in differences in the amount of work-family conflict they experience (Spector et al., 2007). Furthermore the structure of organisations differ resulting in differences in levels of job satisfaction, in particular American firms are often more developmental and rational whereas British firms are often more hierarchical (Lau \& Ngo, 1996). It may therefore be that idiosyncratic factors specific to the US media organisation used in Richardson and Thompson's (2012) study had an influence on the results.

\subsection{The Purpose of the Present Study}

The purpose of the present study is to conduct a comparison of Richardson and Thompson's (2012) study using participants from a British company, a different country and organisational culture to identify potential differences, as well as provide more up to date findings as the fast paced penetration of new technology is resulting in an inability of academic literature to keep up to date. This research will focus primarily on the BB, a new generation Smartphone (Price, 2010) which is widely used within the organisation targeted, and has been reported in recent UK media to have both beneficial and detrimental effects (BBC, 2012; The Guardian, 2013). Previous research has found that BB's can be beneficial to employees as they allow them to continuously monitor and manage information flow even when not 'at work,' enabling them to 'spread the load,' and providing them with a greater sense of control (Allen \& Shoard, 2005; Mazmanian et al., 2006). However it has been noted by Eden $(2001$, p. 187) that 'an urgent threat to respite relief is the high-tech tethers clamped on respitees during their time away from their jobs.' Indeed the telecommunications revolution has resulted in BB's entering the hands of eager users, with employees provided with BB's compulsively checking messages and finding switching off the device difficult even when at home (Mazmanian et al., 2006). This has led to a rise in the term 'Crackberry,' a euphemism becoming Webmaster's Dictionary's 'New word of the year' in 2006 (PRNewswire, 2009).

\subsection{The Study Hypotheses}

This study will investigate the relationship between the usage of the BB device for work purposes during non-work hours and (a) work-family conflict and (b) well-being, and the potential mediators of this relationship with application of the COR theory. Based on past literature and the COR theory predictions the following hypotheses were formed:

Hypothesis 1: $\mathrm{BB}$ use for work purposes during non-work hours will be positively related to work-family conflict and negatively related to well-being. .

Hypothesis 2: The relationship between BB use for work purposes during non-work hours and (a) work-family conflict, and (b) well-being will be mediated by job control.

Hypothesis 3: The relationship between BB use for work purposes during non-work hours and (a) work-family conflict, and (b) well-being will be mediated by detachment from work.

Hypothesis 4: The specific indirect effect of detachment from work as a mediator between BB use for work purposes during non-work hours and (a) work-family conflict, and (b) well-being will be greater than the specific indirect effect of job control as a mediator. 


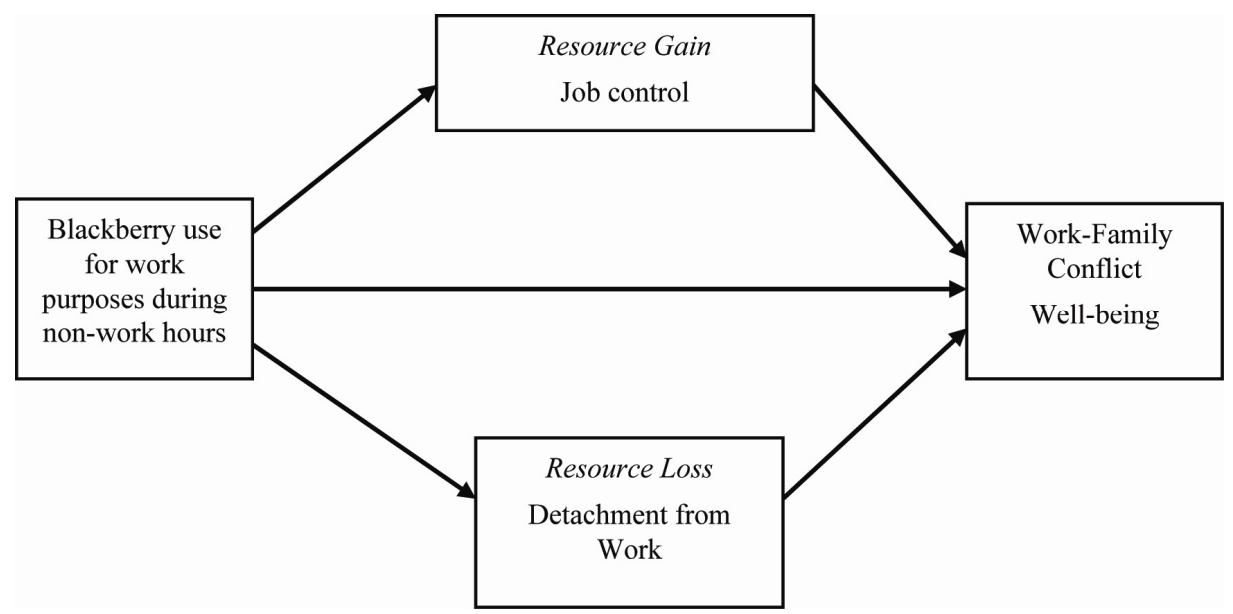

Figure 1. The hypothesized multiple mediation model of BB use for work purposes during non-work hours

\section{Method}

\subsection{Design and Sample}

A correlational survey design was employed to collect data from a convenience sample. There were 90 respondents to the questionnaire, although following data screening only data from 86 participants was analysed. Respondents were working adults based in the UK and employees of one of the world's leading communications service companies who supply their employees with BB devices for work purposes. Both age and gender were controlled for as findings in previous research have suggested they might have an effect on the usage and acceptance of technology (Harrison \& Rainer, 1992). The age of participants ranged from 24-63, with a mean age of 48.60 (SD 8.56), 65 (75.6\%) of which were males and 21 (24.4\%) were females. In addition job level was controlled for as only employees in particular job levels are provided with BB devices within the organisation. Respondents came from two different job levels; senior (53) and junior managers (33).

\subsection{Procedure}

A senior manager within the organisation targeted sent emails to 230 employees which explained the research purpose and provided a URL link to the online questionnaire. To meet the Health and Care Professions Council (HCPC) (2010) and the British Psychological Society (BPS) (2012) guidelines for ethical requirements participants were first directed to participant information and an informed consent checklist, and then debriefed after participation. Participants were also provided with unique usernames and passwords to maintain anonymity. Furthermore respondents were made aware that their participation was voluntary and that they could withdraw their data at any time up until a two week period after the data was collected.

\subsection{Measures}

The beginning of the questionnaire required participants to note their demographic information. Following these questions, a variety of scales were presented to respondents which are described in more detail below. These are scales validated in prior research, with the exception of the duration of BB use measure.

\subsubsection{Blackberry Usage}

To compare the different conceptualizations of BB usage two separate measures were used for frequency and duration. The frequency of BB use for work purposes outside of work hours was measured using the Work Connectivity Behaviour After-Hours (WCBA) frequency measure $(\alpha=.93)$ created by Richardson and Thompson (2012). The measure asks about the use of a specific device, for the purpose of this study this was the BB device, and also how frequently this device is used during a particular non-work activity, such as, during a vacation abroad, party or social event. Items were rated on a 5-point Likert-type scale that ranged from 1 (never) to 5 (always). The duration of BB use during non-work hours for work purposes was measured using items based on the WCBA duration measure $(\alpha=.79)$ created by Richardson and Thompson (2012) which were adapted to fit the present study. For the purpose of the current study participants were asked how much time on average they use their BB to perform job-related duties during non-work hours. Responses were collected for four time periods (before work hours, during break times, after work hours, and during days off) as it was assumed that requesting participants to provide exact minutes would be difficult. In addition due to the boundaries between work and non-work time becoming blurred (Khanna \& Randolph, 2008), it was also assumed that respondents might view 
the terms 'before work' and 'after work' differently therefore an explanation was provided for each time period so that participants were aware of what each period included, such as, 'this includes any time from waking up to before your contracted start time.' The response categories were provided in ranges of minutes to create a likert-type scale, such as, '0 minutes, 1-30 minutes, 31-60 minutes, 61-90 minutes and 91+ minutes.'

\subsubsection{Work-Family Conflict}

Work-family conflict was measured using Kopelman, Greenhaus and Connolly's (1983) 8-item Work Family Conflict scale. Cronbach's alpha reliability coefficients for the 8-item scale range from .78 to .90 (Fields, 2002, p. 201). This particular scale was believed to be ideal for the purpose of the current study as it aims to access the extent of inter-role conflict that occurs between work and family roles, such as, 'my work schedule often conflicts with my family life.' The items were rated on a 5-point Likert-type scale that ranged from 1 (strongly disagree) to 5 (strongly agree).

\subsubsection{Well-Being}

Well-being was measured using the General Health Questionnaire-12 (GHQ-12) (Goldberg, 1972). This questionnaire is a quick, reliable and sensitive short form of the original 60 -item instrument which focuses on the inability to carry out normal functions, as well as the appearance of new disorders or patterns of adjustment that are associated with distress (Goldberg, 1988). It was therefore seen as appropriate for the current study in assessing the current well-being of employees in a sensitive manor, such as, 'have you recently [in the last few weeks] felt constantly under strain?' To make it easier for participants to complete the survey the responses to the questionnaire were altered so that all responses were the same for every question, such as, 'not at all, no more than usual, rather more than usual, much more than usual.' For the purpose of this study the answers were reverse coded so that a high score indicated a high level of well-being.

\subsubsection{Perceived Job Control}

Perceived job control was measured using a 4-item subscale from Kossek et al's (2006) 7-item job control scale ( $\alpha$ $=.74$ ). These four items were assumed to be ideal for the purpose of the current study as they assess an individual's personal flexibility and control in relation to their work, such as, 'In my job, I am able to decide on my own about where the work is done.' To keep the survey format consistent two items were reworded to become statements rather than questions, and so the responses were also changed to match those of the other statements, such as, 'very inaccurate, inaccurate, neither accurate or inaccurate, accurate and very accurate.'

\subsubsection{Psychological Detachment from Work}

Psychological detachment from work was measured using the 4-item psychological detachment subscale of Sonnentag and Fritz's (2007) Recovery Experience Questionnaire. This questionnaire is both valid and reliable in assessing a respondents ability to unwind from work (Sonnentag \& Fritz, 2007), such as, 'during time after work, I forget about work,' therefore is suitable for the present study. Items were rated on a 5-point Likert-type scale that ranged from 1 (strongly disagree) to 5 (strongly agree).

\section{Results}

\subsection{Data Screening}

The data was screened prior to analysis and it was found that some of the data was positively skewed and not normally distributed. In addition using the outlier labelling rule four outliers were identified within the data. These outliers were extreme values from four different participants whose responses to the scales were biased. The data from these participants was removed from the data set as they were considered to not be representative of the whole population. This resulted in a reduction from 90 participants to 86 . Following the removal of these participants the data screening was repeated. The data was normally distributed, independent, and also of an interval level of measurement therefore met the assumptions for parametric testing.

To gain an overview of the frequency of BB usage, a graph was formed showing the activities and events during which employees most frequently use their BB devices for work purposes during non-work hours. 


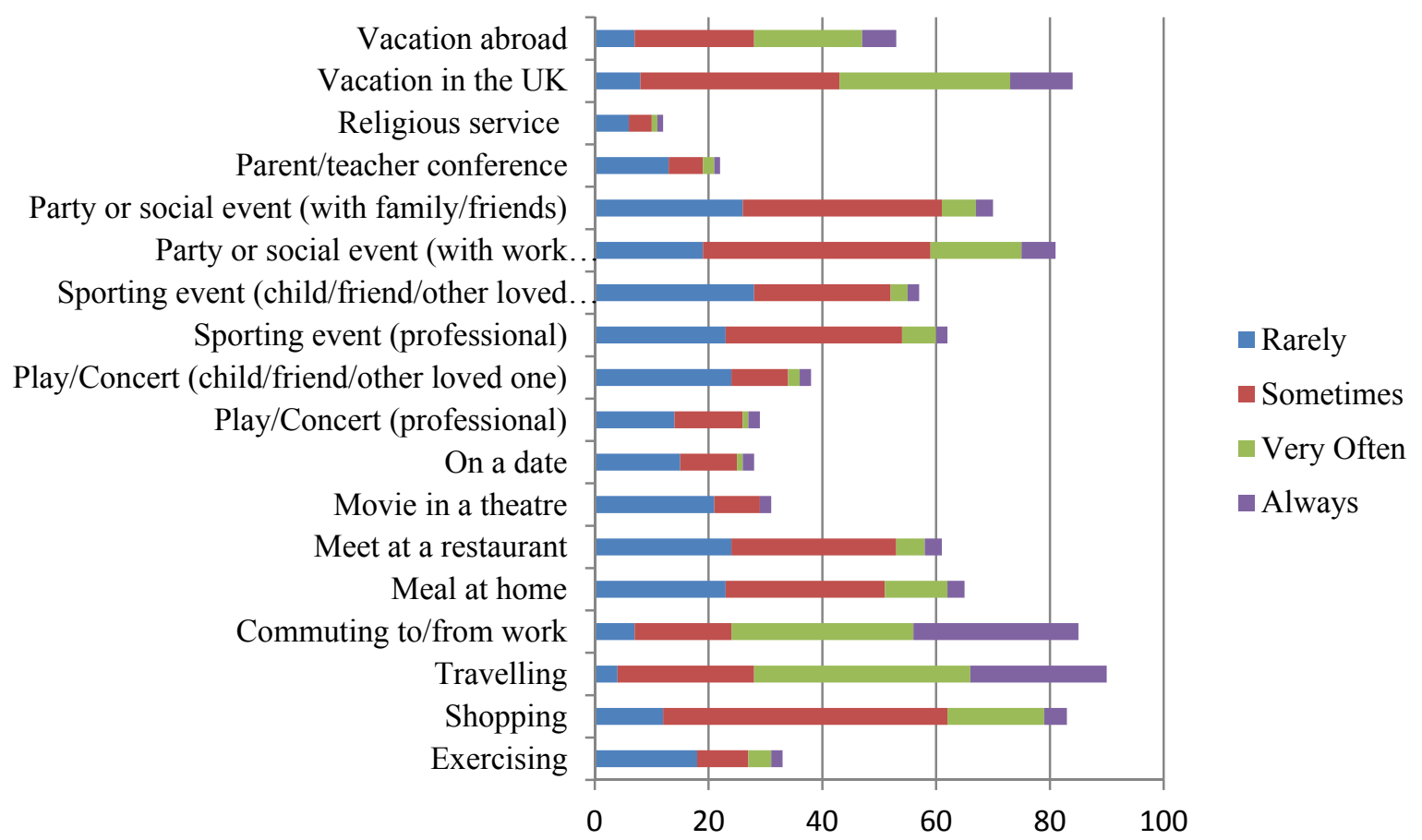

Figure 2. Percentage of participants who frequently use their BB for work purposes during non-work hours for specific activities and events

\subsection{Statistical Analysis}

Table 1 provides the descriptive statistics and correlations among the variables as well as the Cronbach's alpha reliability coefficients for each of the scales which ranged from .73 to .92 indicating high internal consistency.

Table 1. Descriptive statistics and correlations ${ }^{a}$

\begin{tabular}{lccccccccccc}
\hline & Mean & SD & 1 & 2 & 3 & 4 & 5 & 6 & 7 & 8 & 9 \\
\hline 1. Job Level & & & & & & & & & & & \\
2. Age & 48.60 & 8.56 & -.06 & & & & & & & \\
3. Gender & & & .00 & .48 & & & & & & \\
4. Duration of use & 10.17 & 2.74 & -.17 & .01 & -.04 &. $\mathbf{7 3}$ & & & & \\
5. Frequency of use & 40.02 & 9.27 & -.06 & -.12 & -.14 & .66 & $\mathbf{. 8 8}$ & & & \\
6. Work-Family conflict & 27.67 & 6.50 & .04 & .10 & -.04 & .30 & .34 & $\mathbf{. 9 2}$ & & & \\
7. Well-being & 30.72 & 5.24 & -.06 & .02 & .14 & .01 & -.03 & -.41 & $\mathbf{. 8 3}$ & & \\
8. Job control & 14.98 & 2.84 & -.15 & .12 & .12 & -.03 & -.08 & -.36 & .34 &. $\mathbf{7 8}$ & \\
9. Detachment from work & 9.70 & 3.07 & -.11 & -.12 & .17 & -.22 & -.32 & -.70 & .34 & .32 & $\mathbf{. 8 3}$ \\
\hline
\end{tabular}

${ }^{a}$ Correlations greater than 0.30 are significant at the $p<.01$; Correlations greater than 0.21 are significant at the $\mathrm{p}<.05$; The Cronbach's alpha reliability coefficient for each scale appears on the main diagonal in bold.

The bivariate correlations were examined to test Hypothesis 1 . Mixed support was found as work-family conflict was related to both duration $(r=.30, p<.01)$ and frequency of use $(r=.34, p<0.01)$; well-being was not related to duration or frequency of use. The procedures for testing a multiple mediation model stated by Preacher and Hayes (2008) were then followed to investigate the remaining hypotheses, as specifying and testing a multiple mediation model allows for a set of potential mediators to be examined and the 'relative magnitudes of the specific indirect effects associated with all mediators' to be determined (Preacher \& Hayes, 2008, p. 881). Two models were created for each dependent variable (DV) to examine frequency and duration of BB use separately and allow for comparison. To conduct the main analyses an SPSS macro from Preacher and Hayes (2008) was used and bootstrapping procedures were employed to obtain estimates of the indirect effects. 
The two models using work-family conflict as the DV were first examined and were both statistically significant with an $\mathrm{R}^{2}$ value of .5346 for duration of $\mathrm{BB}$ use for work purposes during non-work hours and .5279 for frequency of BB use for work purposes during non-work hours, indicating that the independent variable (IV), mediators and control variables accounted for $53 \%$ and $53 \%$ of the variance in work-family conflict respectively. These findings supported Hypothesis 1 and so further investigation into mediating effects was performed. Table 2 displays the bootstrapped estimates for the total and specific indirect effects for the work-family conflict models.

Table 2. Mediation of the effect of duration and frequency of BB use for work purposes during non-work hours on work-family conflict through job control and detachment from work

\begin{tabular}{lcccc}
\hline Mediator & Bootstrap Estimate & SE & BC 95\% CI Lower & BC 95\% CI Upper \\
\hline Duration Model: & & & & \\
Job Control & .0081 & .0437 & -.0648 & .1173 \\
Detachment from Work & .3266 & .1476 & .0598 & .6441 \\
Total Indirect Effect & .3347 & .1680 & .0236 & .6838 \\
Job control vs. Detachment & -.3185 & .1384 & -.6150 & -.0690 \\
Frequency Model: & & & & \\
Job Control & .0080 & .0116 & -.0071 & .0436 \\
Detachment from Work & .1355 & .0464 & .0521 & .2335 \\
Total Indirect effect & .1435 & .0498 & .0522 & .2472 \\
Job control vs. Detachment & -.1276 & .0459 & -.2244 & -.0454 \\
\hline
\end{tabular}

Note: Based on 5,000 bootstrap samples. $\mathrm{BC}=$ Bias Corrected; $\mathrm{CI}=$ confidence interval

The total indirect effects of BB use for work purposes during non-work hours on work-family conflict through both mediators: job control and detachment from work were then examined. Results were statistically significant for both the duration model $[.3347,95 \%$ BC bootstrap CI $(.0236, .6838)]$ and frequency model $[.1435,95 \% \mathrm{BC}$ bootstrap CI $(.0522, .2472)]$. Tests were then conducted on the significance of the specific indirect effects associated with the two mediators for both models.

The specific indirect effect of BB use for work purposes during non-work hours on work-family conflict through job control was not statistically significant for duration of use $[.0081,95 \%$ BC bootstrap CI $(-.0648, .1173)]$ or for frequency of use $[.0080,95 \%$ BC bootstrap CI $(-.0071, .0436)]$, finding no support for Hypothesis 2 . The direction of the associations between BB usage and job control was also not as predicted in both models. In Model 1 (Figure 3) duration of use was negatively related to job control ( $B=-.03$, ns (Non-significant)), and in Model 2 (Figure 4) frequency of use was also negatively related to job control ( $\mathrm{B}=-.03$, ns). The direction of the associations between job control and work-family conflict in both models was as expected. In Model 1 (Figure 3) job control was negatively related to work-family conflict $(\mathrm{B}=-.36, \mathrm{P}<0.05)$, and in Model 2 (Figure 4) job control was also negatively related to work-family conflict $(B=-.36, n s)$.

The specific indirect effect of BB use for work purposes during non-work hours on work-family conflict through detachment from work was statistically significant for both duration of use [.3266, 95\% BC bootstrap CI $(.0598, .6441)]$ and for frequency of use $[.1355,95 \%$ BC bootstrap CI $(.0521, .2335)]$ providing support for Hypothesis 3. In addition the direction of the associations was as expected in both models. In Model 1 (Figure 3) duration of use was negatively related to detachment from work $(B=-.25, p<.05)$ and detachment from work was negatively related to work-family conflict $(B=-1.30, p<.001)$. In Model 2 (Figure 4) frequency of use was negatively related to detachment from work $(B=-.11, p<.01)$ and detachment from work was negatively related to work-family conflict $(B=-1.29, p<.001)$. 


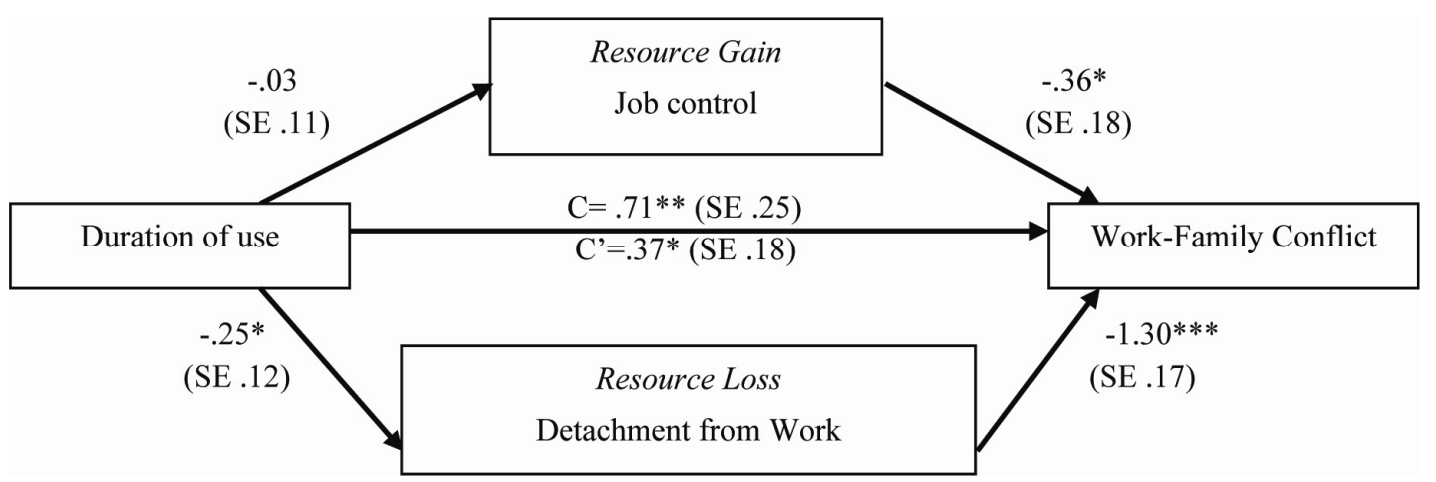

Figure 3. Estimated multiple mediation model based on duration of BB use for work purposes during non-work hours

Note: The numbers in the figure 2 represent the standardized regression coefficients from a bootstrap procedure, $S E=$ standard error, $c=$ total effect, $c^{\prime}=$ direct effect. ${ }^{*} p<.05,{ }^{* *} p<.01,{ }^{* * *} p<.001, \mathrm{R}^{2}=.53$

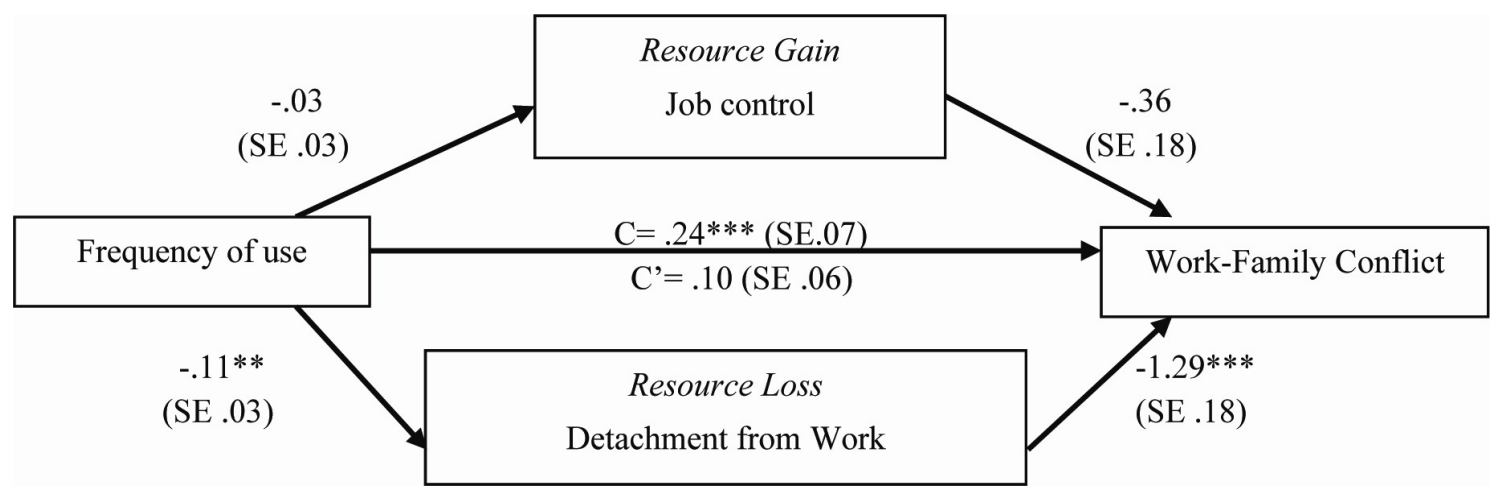

Figure 4. Estimated multiple mediation model based on frequency of BB use for work purposes during non-work hours

Note: The numbers in the figure 3 represent the standardized regression coefficients from a bootstrap procedure, $S E=$ standard error, $c=$ total effect, $c^{\prime}=$ direct effect. ${ }^{*} p<.05,{ }^{* *} p<.01,{ }^{* * *} p<.001, \mathrm{R}^{2}=.53$

The contrast between the specific indirect effect of detachment from work versus the specific indirect effect of job control was found to be statistically significant for both the duration of use model $[-.3185,95 \% \mathrm{BC}$ bootstrap CI $(-.6150,-.0690)]$ and the frequency of use model $[-.1276,95 \%$ BC bootstrap CI $(-.2244,-.0454)]$. This suggests that the specific indirect effect of BB use for work purposes during non-work hours on work-family conflict through detachment from work is larger than the indirect effect of BB use for work purposes during non-work hours on work-family conflict through job control, supporting Hypothesis 4.

The two models using well-being as the DV were then examined, and were also statistically significant with an $\mathrm{R}^{2}$ value of .18 for duration of $\mathrm{BB}$ use for work purposes during non-work hours and .17 for frequency of $\mathrm{BB}$ use for work purposes during non-work hours, indicating that the IV, mediators and control variables accounted for $18 \%$ and $17 \%$ of the variance in well-being respectively. These findings further supported Hypothesis 1 and so further investigation into mediating effects was performed. Table 3 displays the bootstrapped estimates for the total and specific indirect effects for the well-being models. 
Table 3. Mediation of the effect of duration and frequency of BB use for work purposes during non-work hours on well-being through job control and detachment from work

\begin{tabular}{lcccc}
\hline \multicolumn{1}{c}{ Mediator } & Bootstrap Estimate & SE & BC 95\% CI Lower & BC 95\% CI Upper \\
\hline Duration Model: & & & & \\
Job Control & -.0174 & .0576 & -.1693 & .0739 \\
Detachment from Work & -.1133 & .0716 & -.3035 & -.0086 \\
Total Indirect Effect & -.1307 & .1020 & -.3666 & .0456 \\
Job control vs. Detachment & .0959 & .0805 & -.0503 & .2796 \\
Frequency Model: & & & & \\
Job Control & -.0133 & .0169 & -.0647 & .0076 \\
Detachment from Work & -.0482 & .0279 & -.1172 & -.0047 \\
Total Indirect effect & -.0615 & .0317 & -.1323 & -.0081 \\
Job control vs. Detachment & .0349 & .0335 & -.0275 & .1053 \\
\hline
\end{tabular}

Note: Based on 5,000 bootstrap samples. $\mathrm{BC}=$ Bias Corrected; $\mathrm{CI}=$ confidence interval

The total indirect effects of BB use for work purposes during non-work hours on well-being through both mediators: job control and detachment from work were then examined. Results were not statistically significant for the duration of use model $[-.1307,95 \%$ BC bootstrap CI $(-.3666, .0456)]$ but they were for the frequency of use model $[-.0615,95 \%$ BC bootstrap CI $(-.1323,-.0081)]$. Despite the non-significant result for the duration of use model, further tests were still conducted on the significance of the specific indirect effects associated with the two mediators for both models, as significant specific indirect effects are still possible in the presence of a non-significant total indirect effect (Preacher \& Hayes, 2008).

The specific indirect effect of BB use for work purposes during non-work hours on well-being through job control was not statistically significant for duration of use [-.0174, 95\% BC bootstrap CI $(-.1693, .0739)]$ or for frequency of use $[-.0133,95 \%$ BC bootstrap CI $(-.0647, .0076)]$, finding no support for Hypothesis 2 . The direction of the associations between $\mathrm{BB}$ usage and job control was also not as predicted in both models. In Model 1 (Figure 5) duration of use was negatively related to job control $(\mathrm{B}=-.03$, ns), and in Model 2 (Figure 6) frequency of use was also negatively related to job control $(\mathrm{B}=-.03$, ns). The direction of the associations between job control and well-being in both models was as expected. In Model 1 (Figure 5) job control was positively related to well-being $(B=.46, p<.05$ ), and in Model 2 (Figure 6) job control was also positively related to well-being $(B=.46, p<.05)$.

The specific indirect effect of BB use for work purposes during non-work hours on well-being through detachment from work was statistically significant for both duration of use [-.1133, 95\% BC bootstrap CI (-.3035, $-.0086)]$ and for frequency of use [-.0482, 95\% BC bootstrap CI $(-.1172,-.0047)]$ providing support for Hypothesis 3. In addition the direction of the associations was as expected in both models. In Model 1 (Figure 5) duration of use was negatively related to detachment from work $(B=-.25, p<.05)$ and detachment from work was positively related to well-being $(B=.47, p<.05)$. In Model 2 (Figure 6 ) frequency of use was negatively related to detachment from work $(B=-.11, p<.01)$ and detachment from work was positively related to well-being $(B=.47, p<.05)$.

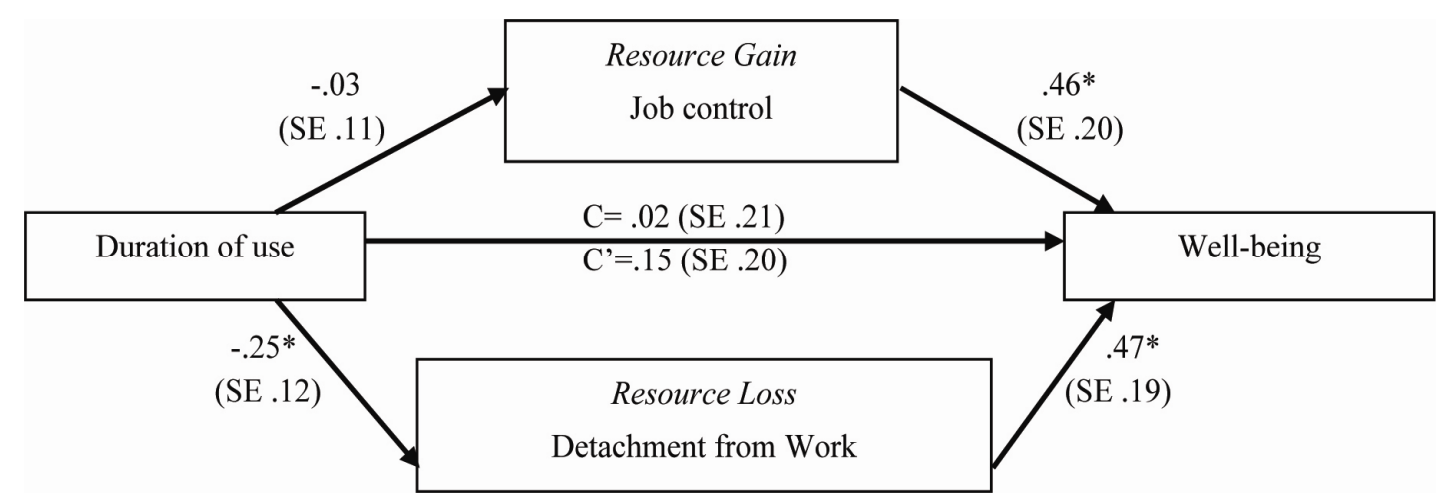

Figure 5. Estimated multiple mediation model based on duration of BB use for work purposes during non-work hours

Note: The numbers in the figure 4 represent the standardized regression coefficients from a bootstrap procedure, $S E=$ standard error, $c=$ total effect, $c^{\prime}=$ direct effect. ${ }^{*} p<.05,{ }^{* *} p<.01,{ }^{* * *} p<.001, \mathrm{R}^{2}=.18$ 


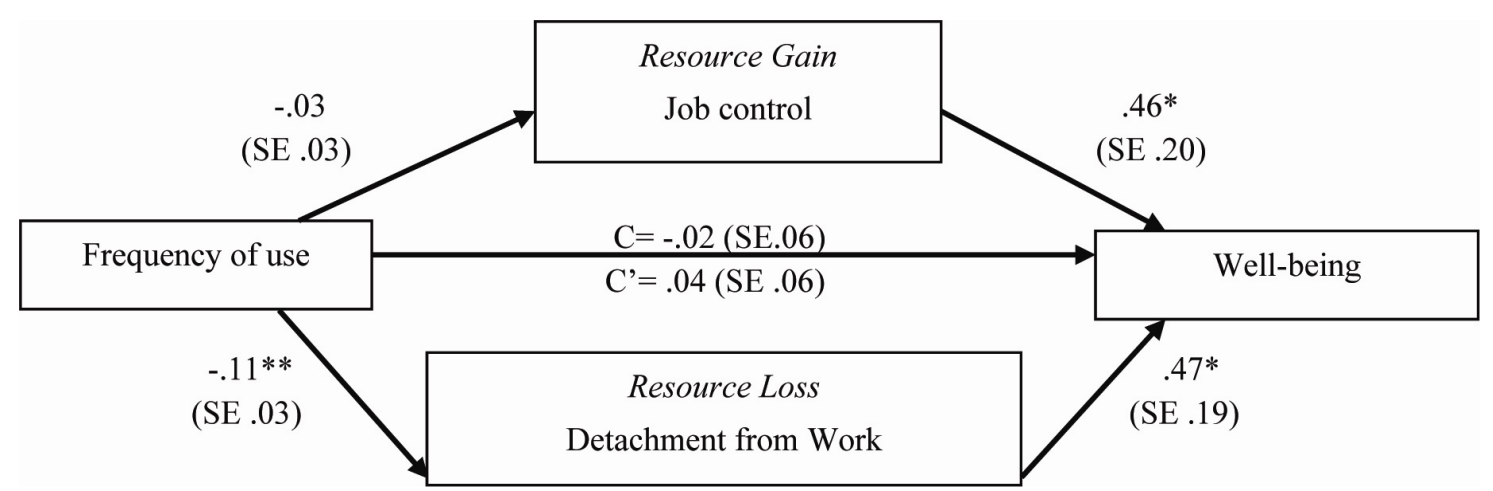

Figure 6. Estimated multiple mediation model based on frequency of BB use for work purposes during non-work hours

Note: The numbers in the figure 5 represent the standardized regression coefficients from a bootstrap procedure, $S E=$ standard error, $c=$ total effect, $c^{\prime}=$ direct effect. ${ }^{*} p<.05,{ }^{* *} p<.01,{ }^{* * *} p<.001, \mathrm{R}^{2}=.17$

The contrast between the specific indirect effect of detachment from work versus the specific indirect effect of job control was examined and found not to be statistically significant for either the duration of use model [.0959, $95 \%$ BC bootstrap CI $(-.0503, .2796)]$ or the frequency of use model $[.0349,95 \%$ BC bootstrap CI $(-.0275, .1053)]$. Therefore the two indirect effects could not be distinguished in terms of magnitude.

\section{Discussion}

This study provided a comparison of Richardson and Thompson's (2012) study using a UK sample, and as predicted it was found that BB use for work purposes during non-work hours was directly related to work-family conflict. In particular high levels of BB use for work purposes during non-work hours was related to higher levels of work-family conflict, supporting hypothesis 1 . The models using work-family conflict as the DV also indicated that the IV, mediators and control variables accounted for $53 \%$ of the variance in work-family conflict, which further supported hypothesis 1 and suggested the need for investigation into the mediating effects. BB use for work purposes during non-work hours was not significantly related to well-being, showing no support for hypothesis 1. As with Richardson and Thompson's (2012) study, the very narrow focus of the GHQ-12 on depression may be the reason for this non-significant finding, as other aspects of well-being may have been more relevant. Furthermore the finding that work-family conflict was strongly correlated with well-being $(\mathrm{r}=-.41)$ suggests that well-being may be further along the causal chain, and so work-family conflict may influence well-being over time. This finding is similar to that of Richardson and Thompson (2012) as they also found a strong correlation $(\mathrm{r}=-.40)$ between work-family conflict and well-being supporting this supposition. Further research is needed using a longitudinal design to test this supposition.

The consistency of these findings with those of Richardson and Thompson (2012) suggest they still hold within a different organisation and culture. An interesting finding in the present study which was not found by Richardson and Thompson (2012) was that despite no significant relationship between BB use for work purposes during non-work hours and well-being, the models using well-being as the DV were statistically significant and indicated that the IV, mediators and control variables accounted for $18 \%$ and $17 \%$ of the variance in well-being, suggesting that further examination was needed into the mediating factors involved.

\subsection{The Mediating Variables}

The findings indicated that job control was not a significant mediator in the relationship between using the BB for work purposes during non-work hours and work-family conflict and well-being for both the duration and frequency model thus did not support hypothesis 2 . These results were surprising given that a variety of past literature found a relationship between using wireless devices and perceptions of control (Allen \& Shoard, 2005; Mazmanian et al., 2006). The results were similar to that of Richardson and Thompson (2012) who found their frequency model which was driven by handheld usage did not find support for the mediation of job control. Allen and Shoard (2005) and Mazmanian et al. (2006) however utilised qualitative methods, specifically interviews which are often associated with more dishonest answers compared to online questionnaire methods due to the presence of a human interviewer (Chang \& Krosnick, 2010). Furthermore these studies investigated the effects of the wireless technology immediately after its introduction to the organisation. In line with Richardson and Thompson's (2012) study the present study did not measure the length of time the employees 
had had their devices. It may be that the initial increase in perception of control straight after the introduction of new technology becomes diminished over time as workload becomes increasingly excessive and employees may gradually become more overwhelmed resulting in a lack of control. This suggests the need for further research into the longitudinal effects of technology use on job control. It is important to note that despite job control not being found to be a mediator, it is still an important factor which should not be ignored. The results showed that job control was positively related to well-being for both models, suggesting that individuals with high levels of job control will also have high levels well-being. Furthermore for the duration model job control was negatively related to work-family conflict, suggesting that individuals with high levels of job control will also be experiencing lower levels of work-family conflict.

The results showed mixed support for hypothesis 3. For the frequency model psychological detachment from work was a significant mediator in the relationship between frequency of BB use for work purposes during non-work hours and work-family conflict. Thus suggesting that employee's who engage frequently with their BB device for work purposes during non-work hours, may be less able to psychologically detach from work, which in turn is related to higher levels of work-family conflict, further supporting the findings of Richardson and Thompson (2012). For the duration model competitive mediation was found as there was both a mediated effect and direct effect but these were in opposite directions. As predicted duration of use was negatively related to detachment from work, and detachment from work was negatively related to work-family conflict. A significant positive direct effect was found between duration of use and work-family conflict, suggesting that psychological detachment from work was not a significant mediator in this relationship. Moreover this identifies an unexplained direct effect and suggests the need for further research to look for an alternative mediator which matches the sign of the revealed direct effect.

The findings also showed that for both the duration and frequency model, psychological detachment from work was a significant mediator in the relationship between BB use for work purposes during non-work hours and well-being. Therefore employees who use their BB's frequently and for long periods of time for work purposes during non-work hours are less likely to psychologically detach from work, which in turn is related to lower levels of well-being. These findings are consistent with those of Sonnentag (2001) who found that not being able to psychologically detach from work during respite results in a lack of stress relief and in turn low levels of well-being. In particular an alarming finding was that $94.2 \%$ of participants reported that at some point during their vacations within the UK they have used their BB devices to engage in work related duties and $58.1 \%$ have done so during vacations abroad. These statistics are significantly greater than those found by Richardson and Thompson (2012) who found that $34 \%$ of participants reported using their wireless devices for work purposes during vacations within the US and abroad, supporting a difference between organisational cultures. When considering the large amount of research that shows that time away from work has a restorative benefit (Eden, 1990; Frankenhaeuser et al., 1989; Westman \& Eden, 1997), these statistics are worrying.

Finally the results indicated that the specific indirect effect of detachment from work was larger than the specific indirect effect of job control for both models using work-family conflict as the DV. Therefore the lack of ability to psychologically detach from work during non-work hours due to increased connectivity to work through the use of the BB device has a greater impact on work-family conflict than job control as a resource-enhancing benefit. This supports hypothesis 4 and the COR theory principle, that resource loss is more salient than resource gain (Freedy \& Hobfoll, 1994; Lee \& Ashforth, 1996).

\subsection{Limitations}

Although the findings discussed above provide new insight they should be interpreted in light of the research limitations. Firstly the ability to assess causality is limited due to the correlational design (Coolican, 2009, p. 452). The multiple mediation regression models suggest that BB use for work purposes during non-work hours precedes job control, detachment from work, work-family conflict and well-being; although the opposite may be true. This therefore suggests that further research is needed to determine causality.

Secondly the use of self report scales may have resulted in response bias, specifically social desirability. In particular the GHQ-12 encompasses some of the most socially undesirable thoughts and feelings such as depression (Hunt, Auriemma, \& Cashaw, 2003), therefore some respondents may attempt to 'look good' by scoring high on well-being and appearing less distressed (Coolican, 2009, p. 183). This may be the reason for no significant relationship being identified between BB use for work purposes during non-work hours and well-being. It may be ideal for future researchers therefore to collect more objective data such as heart rate and blood pressure as well as employee records of time away from work due to illness, in particular work-related 
stress. Moreover investigating the perceptions of family members may also be useful in indentifying if employees reports of their general well-being are consist with that of their families observations.

Thirdly, although participants were from a worldwide organisation, respondents were those based only in the UK, therefore as with Richardson and Thompson's (2012) study the findings may not be generalisable to other populations due to differences across countries (Lau \& Ngo, 1996; Spector et al., 2007). Furthermore the results may not be generalisable to other organisations as they differ considerably in there assumptions about human relationships, time, space, social support and control, which in turn results in differences in work-related stress and illness (Peterson \& Wilson, 2002). It may be that idiosyncratic factors specific to the organisation in the present study had an influence on the results. For example, the expectations to connect to the workplace during non-work hours may be higher or lower within this organisation compared to others. Therefore a cross-cultural study using various organisations should be conducted. Although this research did find similar results to Richardson and Thompson (2012) showing similarities between the US and the UK, as well as different organisations.

\subsection{Practical Applications}

Despite the limitations of the current research it does have good practical application. The findings suggest that organisations need to recognise the negative impact that increasing use of the BB device for work purposes during non-work hours is having on employees, especially as work-family conflict is a major problem for both individuals and organisations, reducing productivity (Bilal, Zia-ur-Rehman, \& Raza, 2010) and employee life satisfaction (Carlson \& Perrewé, 1999), and increasing psychological distress, somatic complaints (Frone et al., 1992, p. 197), absenteeism and turnover (Bilal et al., 2010). It is proposed that organisations should aim to reduce this impact by ensuring employees are able to detach from work during time away from work. This could be done by reducing the amount of time needed to be connected to these devices, through providing more manageable workloads, which in turn may increase an individual's perception of control. It is anticipated that minimising the use of these devices will be problematic for organisations and it is unlikely that these devices will disappear from organisational life. It is therefore important to find alternative ways of reducing their impact. One way in which this could be done is by moving the focus onto individual employees. As previously discussed communications technologies do not force the user to stay connected, it is the user's choice as to how they take on, negotiate and accept the technology (Silverstone \& Mansell, 1996). Therefore employer's need to make sure employees are able to effectively use these devices as their ability to use them will impact on both the individual and the organisation as a whole.

One way to help ensure employees are effectively able to use these devices is by taking into account individual personalities and abilities during the recruitment process. Candidates should be made aware that a job entails a heavy workload and is likely to result in greater demands of time and resources (Department of Health, 2007). Sufficient psychometric selection tests should be used in order for employers to identify whether the candidate will be able to cope with such demands (Cheminais, Van Der Waldt, Bayat, \& Fox, 1998, p. 140-141). It has been suggested in previous research that some individuals develop and maintain their own boundaries to separate work and non-work domains (Ashforth, Kreiner, \& Fugate, 2000), a 'mental framework' which they idiosyncratically construct (Nippert-Eng, 1996a; b). These individuals may be more suitable for a job encompassing high level demands.

Furthermore to help current employees, employers could administer training and education programmes which can help to enhance employee skills (The Society for Human Resource Management, 2008), and improve mental health and well-being (Department of Health, 2007). In particular it is important employers make employees aware of issues which may impact on well-being, including excessive use of their BB device for work purposes during non-work hours. It can also be beneficial to make suggestions on how best to psychologically detach from work demands and establish a healthy work-life balance, as well as educating employees on how to recognise the early signs or symptoms of decreasing mental health and well-being, and providing information about where to seek support (Department of Health, 2007). This investment of time from the employer will not only help the employee but also benefit themselves in terms of engagement and motivation, and also the organisation as a whole, increasing productivity and reducing absenteeism (Department of Health, 2007). It is important to note that employers should also be provided with training to help employees. Recent research found that only $13 \%$ of line managers had received training on mental health awareness, and many reported that this training was not sufficient and that continuous information and support would be more useful (The Great Place to Work Institute, 2008).

\section{Conclusion}

The present research suggests that employees who engage with their BB devices for work purposes during non-work hours experience higher levels of work-family conflict. In particular those who use the device 
frequently will not psychologically detach from work, which is related to work-family conflict and lower levels of well-being. Furthermore those who use the device for long periods of time will also not psychologically detach from work, which is related to lower levels of well-being. The research did not support an employee's perception of control as a mediator; although there were interesting findings which suggested job control may be a potential benefit to employees. The findings did support the COR theory, finding that the mediating effect of detachment from work on the relationship between BB use and work-family conflict was greater than the mediating effect of job control, showing that resource loss is more salient than resource gain. Despite the limitations acknowledged in the current research there were a variety of practical applications. The report proposes that organisations should aim to reduce the negative impact the usage of the BB device for work purposes during non-work hours has on employees, by either reducing the usage of this device or by focusing on enhancing the skills and abilities of employees, ensuring they are capable of handling the device and demands of the job. Further research is needed into new communication technologies such as the BB and whether the overall consequences of usage are positive or negative for both employees and the organisation. Moreover investigation should be undertaken into the unexplained direct effect to identify an alternative mediator in the relationship between duration of BB use for work purposes during non-work hours and work-family conflict.

\section{Acknowledgements}

I am grateful to many who have provided feedback and assistance throughout this project. In particular I am sincerely grateful to my supervisor, whose support, guidance and encouragement throughout has made this research possible. In addition I would like to show my gratitude to the organisation who contributed to this report, and also my family and friends who have provided unlimited support and boosted my morale.

This report is based on the Undergraduate dissertation of S. Ward, presented in Faculty of Health and Life Sciences, Psychology and Behavioural Sciences Department, Coventry University, Coventry, UK, towards the degree of a Bachelor of Science with Honours in Psychology.

\section{References}

Allen, D. K., \& Shoard, M. (2005). Spreading the load: Mobile information and communications technologies and their effect on information overload. Information Research, 10(2), 227.

Ashforth, B., Kreiner, G., \& Fugate, M. (2000). All in a day's work: Boundaries and micro role transitions. Academy of Management Review, 25(3), 472-491. http://dx.doi.org/10.2307/259305

BBC. (2012). Is teleworking driving us crazy? Retrieved from http://www.bbc.co.uk/news/business- 19594518

Bélanger, F. (1999). Workers' propensity to telecommute: An empirical study. Information and Management, 35(3), 139-153. http://dx.doi.org/10.1016/S0378-7206(98)00091-3

Bilal, M., Zia-ur-Rehman, M., \& Raza, I. (2010). Impact of Family Friendly Policies on Employees' Job Satisfaction and Turnover Intention (A study on work-life balance at workplace). Interdisciplinary Journal of Contemporary Research in Business, 2(7), 378.

Braunstein-Bercovitz, H., Frish-Burstein, S., \& Benjamin, B. A. (2012). The role of personal resources in work-family conflict: Implications for young mothers' well-being. Journal of Vocational Behaviour, 80(2), 317-325. http://dx.doi.org/10.1016/j.jvb.2011.10.003

Carlson, D. S., \& Perrewé, P. L. (1999). The role of social support in the stress or-strain relationship: An examination of work-family conflict. Journal of Management, 25(4), 513-540. http://dx.doi.org/10.1177/014920639902500403

Chang, L., \& Krosnick, J. A. (2010). Comparing oral interviewing with self-administered computerised questionnaires: An experiment. Public Opinion Quarterly, 74(1), 154-167. http://dx.doi.org/10.1093/poq/nfp090

Cheminais, J., Van Der Waldt, G., Bayat, M. S., \& Fox, W. (1998). The Fundamentals of Public Personnel Management. Kenwyn: Juta and Company Ltd.

Coolican, H. (2009). Research Methods and Statistics in Psychology (5th ed.) London: Hodder and Stoughton.

De Jonge, J., Janseen, P. P. M., \& van Breukelen, G. J. P. (1996). Testing the demand-control-support model among health-care professionals: A structural equation model. Work and Stress: An International Journal of Work, Health and Organisations, 10(3), 209-224. http://dx.doi.org/10.1080/02678379608256801 
Department of Health. (2007). Line Managers Resource: A Practical Guide to Managing and Supporting People with Mental Health Problems in the Workplace. Retrieved from http:/www.hse.gov.uk/stress/pdfs/manage-mental-health.pdf

Eden, D. (1990). Acute and chronic job stress, strain, and vacation relief. Organizational Behaviour and Human Decision Processes, 45(2), 175-193. http://dx.doi.org/10.1016/0749-5978(90)90010-7

Eden, D. (2001). Job stress and respite relief: Overcoming high-tech tethers. Exploring Theoretical Mechanisms and Perspectives, 1, 143-194. http://dx.doi.org/10.1016/S1479-3555(01)01012-5

Fields, D. L. (2002). Taking the Measure of Work: A Guide to Validated Scales for Organizational Research and Diagnosis. California: Sage Publications, Inc.

Frankenhaeuser, M., Lundberg, U., Fredrikson, M., Melin, B., Tuomisto, M., Myrstern, A., ... Willin, L. (1989) 'Stress on and off the job as related to sex and occupational status in white-collar workers.' Journal of Organizational Behaviour, 10(4), 321-346. http://dx.doi.org/10.1002/job.4030100404

Freedy, J. R., \& Hobfoll, S. E. (1994). Stress inoculation for reduction of burnout: A conservation of resources approach. Anxiety, Stress, and Coping, 6, 311-325. http://dx.doi.org/10.1080/10615809408248805

Freudenberger, H. J. (1974). Staff burn-out. Journal of Social Issues, 30(1), 159-164. http://dx.doi.org/10.1111/j.1540-4560.1974.tb00706.x

Frissen, V. A. J. (2000). ICTs in the Rush Hour of Life. The Information Society, 16(1), 65-75. http://dx.doi.org/10.1080/019722400128338

Frone, M. R., Russell, M., \& Cooper, M. L. (1992). Antecedents and outcomes of work-family conflict: Testing a model of work-family interface. Journal of Applied Psychology, 77(1), 65-78. http://dx.doi.org/10.1037//0021-9010.77.1.65

Goldberg, D. (1972). The detection of psychiatric illness by questionnaire: A technique for the identification and assessment of non-psychotic psychiatric illness. London, New York: Oxford University Press.

Goldberg, D. P., \& Williams, P. (1988). A user's guide to the General Health Questionnaire. Windsor UK: NFER-Nelson.

Greenhaus, J. H., \& Beutell, N. J. (1985). Sources of conflict between work and family roles. Academy of Management Review, 10(1), 76-88. http://dx.doi.org/10.2307/258214

Harrison, A. W., \& Rainer, R. K. Jr. (1992). The influence of individual differences on skill in end-user computer. Journal of Management Information Systems, 9(1), 93-111.

Health and Care Professions Council. (2010). Information for students and education providers: Guidance on conduct and for fhics students. Retrieved from http://www.hpc-uk.org/assets/documents/10002C16Guidanceonconductandethicsforstudents.pdf

Hill, J. E., Hawkins, A. J., Ferris, M., \& Weitzman, M. (2001). Finding an Extra Day a Week: The Positive Influence of Perceived Job Flexibility on Work and Family Life Balance. National Council on Family Relations, 50(1), 49-58. http://dx.doi.org/10.1111/j.1741-3729.2001.00049.x

Hobfoll, S. E. (1988). The ecology of stress. Washington, DC: Hemisphere.

Hobfoll, S. E. (1989). Conservation of resources: A new attempt at conceptualizing stress. American Psychologist, 44(3), 513-524. http://dx.doi.org/10.1037//0003-066X.44.3.513

Hobfoll, S. E. (1998). Stress, Culture, and Community: The Psychology and Philosophy of Stress. New York: Plenum Press.

Hobfoll, S. E. (2001). The influence of culture, community, and the nested-self in the stress process: Advancing conservation of resources theory. Applied Psychology: An International Review, 50(3), 337-421. http://dx.doi.org/10.1111/1464-0597.00062

Hobfoll, S. E., \& Freedy, J. (1993). Conservation of resources: A general stress theory applied to burnout. In Schaufeli, W. B., Maslach, C., \& Marek, T. (Eds.) Professional burnout: Recent developments in theory and research (pp. 115-129). Washington, DC: Taylor \& Francis.

Hunt, M., Auriemma, J., \& Cashaw, A. C. A. (2003). Self-Report Bias and Underreporting of Depression on the BDI-II. Journal of Personality Assessment, 80(1), 26-30. http://dx.doi.org/10.1207/S15327752JPA8001_10 
Ilies, R., Schwind, K. M., Wagner, D. T., Johnson, M. D., DeRue, D. S., \& IIgen, D. R. (2007). When Can Employees Have a Family Life? The Effects of Daily Workload and Affect on Work-Family Conflict and Social Behaviors at Home. Journal of Applied Psychology, 92(5), 1368-1379. http://dx.doi.org/10.1037/0021-9010.92.5.1368

Jarvenpaa, S. L., \& Lang, K. R. (2005). Managing the paradoxes of mobile technology. Information Systems Management, 22(4), 7-23. http://dx.doi.org/10.1201/1078.10580530/45520.22.4.20050901/90026.2

Karatepe, O. M., Sokmen, A., Yavas, U., \& Babakus, E. (2010). Work-family Conflict and Burnout in Frontline Service Jobs: Direct, Mediating and Moderating Effects. E + M Economics and Management Journal, 13(4), 61-73.

Khanna, S., \& Randolph, J. (2008). Revolutionising the Workplace: A Case Study of the Future of Work Program at Capital One. Human Resource Management, 47(4), 795-808. http://dx.doi.org/10.1002/hrm.20245

Kopelman, R. E., Greenhaus, J. H., \& Connolly, T. F. (1983). A model of work, family, and interrole conflict: A construct validation study. Organizational Behavior and Human Performance, 32, 198-215. http://dx.doi.org/10.1016/0030-5073(83)90147-2

Kossek, E. E., Lautsch, B. A., \& Eaton, S. C. (2006). Telecommuting, control, and boundary management: Correlates of policy use and practice, job control, and work-family effectiveness. Journal of Vocational Behaviour, 68(2), 347-367. http://dx.doi.org/10.1016/j.jvb.2005.07.002

Lau, C-M., \& Ngo, H-Y. (1996). One County Many Cultures: Organizational Cultures of Firms of Different $\begin{array}{lllll}\text { Country Origins. International Business } & \text { Review, }\end{array}$ http://dx.doi.org/10.1016/0969-5931(96)00022-4

Lee, R. T., \& Ashforth, B. E. (1996). A meta-analytic examination of the correlates of the three dimensions of job burnout. Journal of Applied Psychology, 81(2), 123-133. http://dx.doi.org/10.1037//0021-9010.81.2.123

Maslach, C. (1982). Burnout: The cost of caring. Englewood Cliffs, NJ: Prentice Hall.

Mazmanian, M., Orlikowski, W. J., \& Yates, J. (2006). Ubiquitous email: Individual experiences and organizational consequences of BlackBerry use. Paper presented at the Annual Academy of Management Conference, Atlanta, GA.

Nippert-Eng, C. E. (1996a). Calendar and Keys: The classification of "home" and "work". Sociological Forum, 11(3), 563-582.

Nippert-Eng, C. E. (1996b). Home and work: Negotiating boundaries through everyday life. Chicago: The University of Chicago Press.

O'Driscoll, M., Brough, P., \& Kalliath, T. (2006). The changing nature of work and its impact on the work-home interface. In Jones, F., Burke, R. J., \& Westman, M. (2006). Work-Life Balance, a Psychological Perspective (pp. 14-38). Hove: Psychology Press.

Ofcom. (2012). Communications Market Report. Retrieved from http://stakeholders.ofcom.org.uk/binaries/research/cmr/cmr12/CMR_UK_2012.pdf

Peterson, M., \& Wilson, J. F. (2002). The Culture-Work-Health Model and Work Stress. American Journal of Health Behaviour, 26(1), 16-24. http://dx.doi.org/10.5993/AJHB.26.1.2

Preacher, K. J., \& Hayes, A. F. (2008). Asymptotic and resampling strategies for assessing and comparing indirect effects in multiple mediator models. Behaviour Research Methods, 40(3), 879-891. http://dx.doi.org/10.3758/BRM.40.3.879

Price, I. (2010). "Email is ruining my life!” What is the impact of doing work email via BlackBerry on stress? A quantitative study. Retrieved from http://www.grimsdykeconsulting.com/wp-content/uploads/2010/07/Ian-Price-Research-Project-final.pdf

PRNewswire. (2006). Webster's New World(R) College Dictionary: Info Addicts Are All Thumbs. Retrieved from $\mathrm{http} / / / \mathrm{www}$.prnewswire.com/news-releases/websters-new-worldr-college-dictionary-info-addicts-are-all-thu mbs-55824847.html

Richardson, K. M., \& Thompson, C. A. (2012). High Tech Tethers and Work-family Conflict: A conservation of Resources Approach. Engineering Management Research, 1(1), 29-43. http://dx.doi.org/10.5539/emr.v1n1p29 
Ross, C. E., \& Reskin, B. F. (1992). Education, control at work, and job satisfaction. Social Science Research, 21(2), 134-148. http://dx.doi.org/10.1016/0049-089X(92)90012-6

Schaufeli, W. B., \& Bakker, A. B. (2004). Job demands, job resources, and their relationship with burnout and engagement: A multi-sample study. Journal of Organizational Behaviour, 25(3), 293-315. http://dx.doi.org/10.1002/job.248

Silverstone, R., \& Mansell, R. (1996). Communication by Design: The Politics of Information and Communication Technologies. Oxford: Oxford University Press.

Sonnentag, S. (2001). Work, recovery activities, and individual well being: A diary study. Journal of Occupational Health Psychology, 6(3), 196-210. http://dx.doi.org/10.1037//1076-8998.6.3.196

Sonnentag, S., \& Bayer, U.V. (2005). Switching off mentally: Predictors and consequences of psychological detachment from work during off-job time. Journal of Occupational Health Psychology, 10(4), 393-341. http://dx.doi.org/10.1037/1076-8998.10.4.393

Sonnentag, S., \& Fritz, C. (2007). The recovery experience questionnaire: Development and validation of a measure for assessing recuperation and unwinding from work. Journal of Occupational Health Psychology, 12(3), 204-221. http://dx.doi.org/10.1037/1076-8998.12.3.204

Spector, P. E., Allen, T. D., Poelmans, S. A. Y., Lapierre, L. M., Cooper, C. L., O’Driscoll, M., ... Widerszal-Bazyl, M. (2007). Cross-national differences in relationships of work demands, job satisfaction, and turnover intentions with work-family conflict. Personal Psychology, 60(4), 805-835. http://dx.doi.org/10.1111/j.1744-6570.2007.00092.x

The British Psychological Society. (2012). Psychological Testing a User's Guide. Retrieved from http://www.psychtesting.org.uk/download\$.cfm?file_uuid=D6010DB1-A98D-DDBC-781B-FA401185DB B8\&siteName $=$ ptc

The Great Place to Work Institute. (2008). Beyond Stress: A Guide to Mental Health and Workplace Wellbeing. Retrieved from http://base-uk.org/sites/base-uk.org/files/\%5Buser-raw\%5D/11-07/beyond-stress-a-guide-to-mental-healthand-workplace-wellbeing.pdf

The Guardian. (2013). The Rise of the 24-hour-email culture: The Blackberry isn't called the crackberry for nothing. Retrieved from http://careers.guardian.co.uk/careers-blog/blackberry-equals-no-work-life-balance

The Society for Human Resource Management. (2008). Critical Skills Needs and Resources for the Changing Workforce: Keeping Skills Competitive. Retrieved from http://www.shrm.org/research/surveyfindings/articles/documents/critical\%20skills\%20needs\%20and\%20re sources $\% 20$ for $\% 20$ the $\% 20$ changing $\% 20$ workforce $\% 20$ survey $\% 20$ report.pdf

Thompson, C. A., \& Prottas, D. J. (2006). Relationships among organizational family support, job autonomy, perceived control, and employee well being. Journal of Occupational Health Psychology, 11(1), 100-118. http://dx.doi.org/10.1037/1076-8998.10.4.100

Van Yperen, N. W., \& Hagedoorn, M. (2003). Do High Job Demands Increase Intrinsic Motivation or Fatigue or Both? The Role of Job Control and Job Social Support. Academy of Management Journal, 46(3), 339-348. http://dx.doi.org/10.2307/30040627

Vendramin, P., Valenduc, G., Rolland, I., Richardson, R., Gillespie, A., Belt, V., Carré, D., Maugéri, S., Combès, Y., Ponzellini, A., Pedersini, R., \& Neri, S. (2000). Flexible Work Practices and Communication Technology: Flexcot. A Report for the European Commission. Retrieved from http://www.ftu-namur.org/fichiers/FLEXCOT-rapfinal.pdf

Westman, M., \& Eden, D. (1997). Effects of vacation on job stress and burnout: Relief and fade-out. Journal of Applied Psychology, 82(4), 516-527. http://dx.doi.org/10.1037/0021-9010.82.4.516

\section{Copyrights}

Copyright for this article is retained by the author(s), with first publication rights granted to the journal.

This is an open-access article distributed under the terms and conditions of the Creative Commons Attribution license (http://creativecommons.org/licenses/by/3.0/). 